\title{
Surface Enhanced Raman Scattering of Carbon Nanotubes Decorated by Individual Fluorescent Gold Particles
}

\author{
Matteo Scolari, ${ }^{\dagger}$ Alf Mews, ${ }^{* \dagger}$ Nan Fu ${ }^{\dagger}$ Anton Myalitsin, ${ }^{\dagger}$ Tilman Assmus,, \\ Kannan Balasubramanian, ${ }^{\ddagger}$ Marko Burghard, ${ }^{\ddagger}$ and K. Kern $\$, \S$ \\ Department of Chemistry, University of Siegen, D-57068 Siegen, Germany, Max Planck Institute for \\ Solid State Research, D-70569 Stuttgart, Germany, and EPFL, CH-1015 Lausanne, Switzerland
}

Received: August 2, 2007; In Final Form: October 10, 2007

\begin{abstract}
Confocal optical microscopy was employed to study the effect of surface-enhanced Raman scattering on individual single-walled carbon nanotubes covered with isolated gold particles. The gold particles with diameters between 10 and $120 \mathrm{~nm}$ were deposited in low densities on the tubes' sidewalls by an electrochemical method. In the spectra, Raman peaks associated with the nanotubes were found to be superimposed on a broad luminescence background originating from the metal particles. With increasing particle size, both the luminescence intensity as well as the Raman enhancement increased at longer wavelengths. This finding is consistent with a size-dependent broadening of the gold plasmon frequency and a corresponding extension of the energetic range for local field enhancement on the particle surface. In addition, wavelength-dependent experiments revealed a maximum Raman intensity when both nanotube and metal particle were in optical resonance.
\end{abstract}

\section{Introduction}

Progress in nanoscience critically depends on the ability to controllably tune the electronic and optical properties of nanostructures. This task can be achieved through the variation of size and shape of the respective materials or by combining different nanostructures, from which useful cooperative effects can evolve. Of particular interest is the combination of singlewalled carbon nanotubes (SWNTs) and gold nanoparticles (NPs), which represent two of the most intensively studied nanoscopic systems whose properties are sensitive to the chemical environment. ${ }^{1}$

For isolated SWNTs, it has been demonstrated that the electronic properties do not only depend on their structure ${ }^{2}$ but can also be modulated by chemical modifications such as doping $^{3}$ or the attachment of functional groups ${ }^{4}$ or metal nanoparticles. ${ }^{5,6}$ Progress along these lines has led to the successful realization of devices such as chemical sensors, ${ }^{7}$ rectifying diodes, ${ }^{8}$ and single-electron transistors. ${ }^{9}$ In this context, the evaluation of the impact of the appended moieties on the tube's properties strongly relies upon characterization methods applicable at the single nanotube level, which include Raman and fluorescence spectroscopy, ${ }^{10,11}$ scanning tunneling microscopy ${ }_{12}$ transmission electron microscopy,${ }^{13}$ as well as combinations of spectroscopic and microscopic techniques. ${ }^{14}$ Similar methods have been used to characterize noble metal nanoparticles, ${ }^{15}$ which display a distinct, size-dependent optical absorption associated with the excitation of surface plasmons involving the collective oscillation of the free electrons. ${ }^{16}$ Besides the plasmon absorption, such nanocrystals also exhibit weak luminescence. ${ }^{17,18}$ The phenomenon of gold fluorescence was first observed by Mooradian ${ }^{19}$ and assigned to the radiative

\footnotetext{
* Corresponding author. E-mail: alf.mews@uni-siegen.de.

$\dagger$ University of Siegen.

Max Planck Institute for Solid State Research.

$\S$ EPFL.
}

recombination of electrons from the 6sp-band close to the Fermi level with holes in the $5 \mathrm{~d}$-band. ${ }^{20}$ While the photoluminescence quantum yield (PL-QY) is only on the order of $10^{-10}$ for bulk gold, it increases for small particles if the energy range of the plasmon coincides with the interband recombination. For gold nanocrystals, the PL-QY can be up to $10^{-4}$ as a consequence of the strong local electric field enhancement caused by the surface plasmon. ${ }^{21,22}$

While the structure-property relationships of both isolated SWNTs and isolated metal NPs have been intensively explored, much less is known about corresponding hybrids. In the present work, we investigated the local spectroscopic properties of individual SWNTs decorated with gold nanoparticles. The particles were deposited onto the tubes via an electrochemical approach, whereby an intimate physical contact between the two components is ensured. This renders the SWNT/NP hybrids into a suitable test bed for studying surface-enhanced Raman scattering (SERS), which mainly arises from enhanced optical fields in the vicinity of the NPs. The near field of metal particles has enabled vibrational spectroscopy down to the singlemolecule detection limit. ${ }^{23}$ Such experiments, however, were often hampered by a poorly defined position and orientation of the molecules with respect to the metal structure, which might even change during investigation. ${ }^{24} \mathrm{By}$ contrast, the present welldefined configuration offers the advantages of high stability even under strong illumination and the possibility to examine the same nanoscopic objects with different excitation laser wavelengths. Moreover, it allows for a clear assignment of the spectral features and to evaluate their dependence on the nanoparticle size, representing an important step toward quantification of the surface-enhanced resonance Raman scattering (SERRS) effect.

\section{Experimental Procedures}

The carbon nanotubes investigated in this work were synthesized by chemical vapor deposition (CVD) growth with iron 

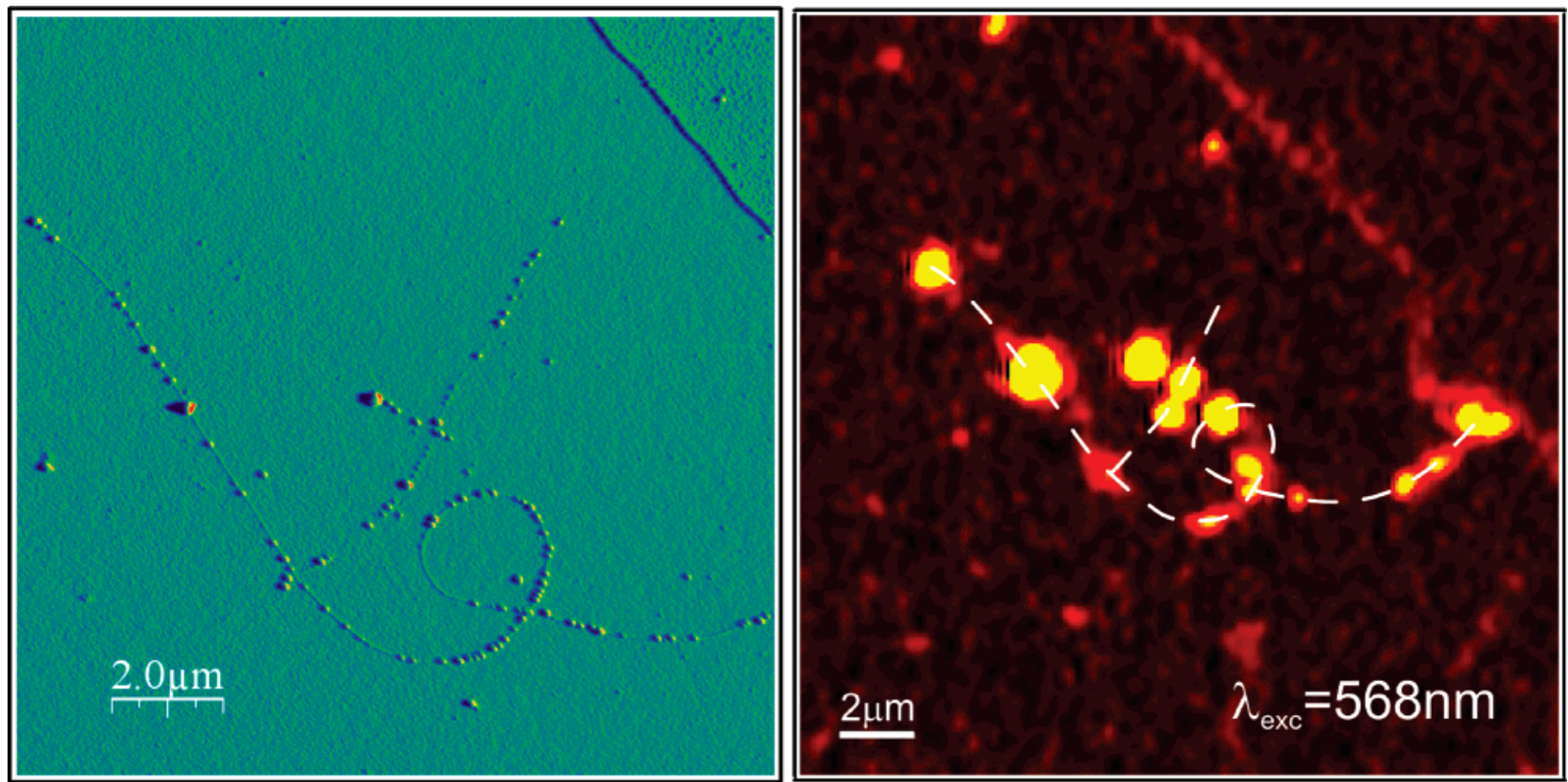

Figure 1. AFM (left) and Raman G-line image (right) of a loop-shaped, gold nanoparticle-decorated SWCNT (highlighted by a dashed white line in the right panel). The emitted photoluminescence in the Raman image is associated with gold nanoparticles in resonance with the excitation wavelength $\left(\lambda_{\text {exc }}=568 \mathrm{~nm}\right)$.

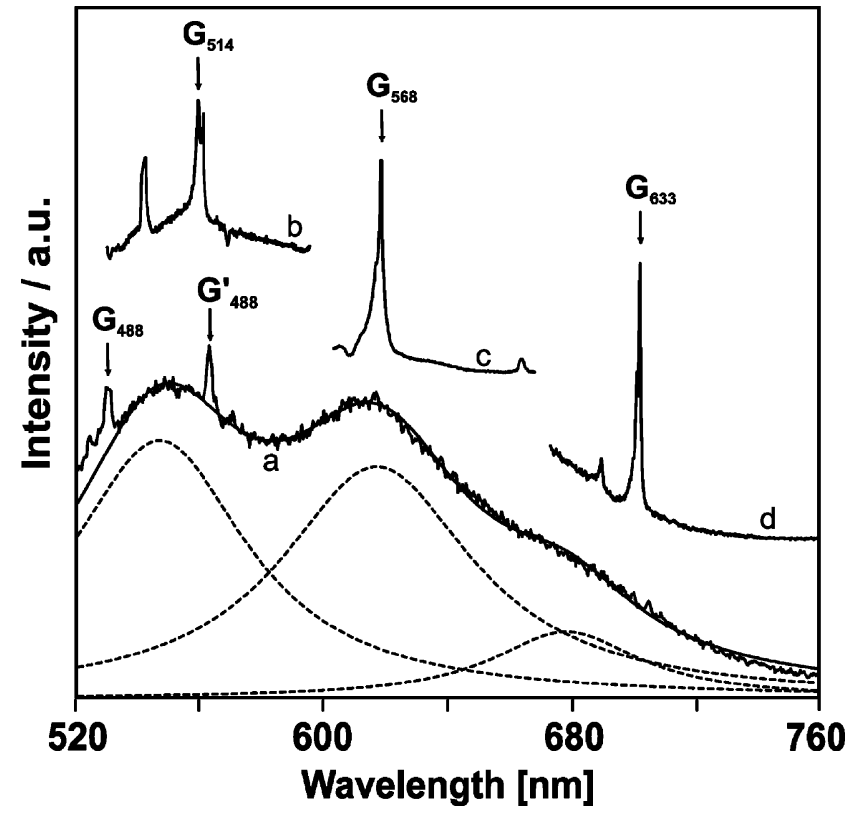

Figure 2. Selection of different emission spectra obtained from a 90 nm gold nanoparticle grown on a SWNT. The spectra were acquired with different excitation wavelengths: $488 \mathrm{~nm}$ (a), $514 \mathrm{~nm}$ (b), 568 $\mathrm{nm}(\mathrm{c})$, and $633 \mathrm{~nm}(\mathrm{~d})$. The wavelength-dependent Raman modes (Gand $\mathrm{G}^{\prime}$-lines) are superimposed on a broad fluorescence background that can be fitted with three Lorentzian peaks (dashed lines).

nanoparticles as the catalyst and ethanol as the carbon feedstock. The SWNTs were contacted with AuPd (60:40) electrodes (15 nm thickness) through standard e-beam lithography. Afterward, the samples were subjected to thermal annealing at $250{ }^{\circ} \mathrm{C}$ for 60 min under an argon atmosphere. Electrodeposition of gold onto the tubes was accomplished inside an electrochemical cell containing an aqueous solution ( $\mathrm{pH} 2)$ with $2 \mathrm{mM} \mathrm{KAuCl}_{4}, 5$ $\mathrm{mM}$ poly $N$-vinyl-2-pyrrolidone (PVP K30, Aldrich) as the stabilizer, and $0.1 \mathrm{mM} \mathrm{LiCl}_{4}$ as the supporting electrolyte. A tungsten needle was used as the working electrode, while platinum wires were used as counter and reference electrodes. Electrodeposition performed with a potential of $-0.8 \mathrm{~V}$ applied

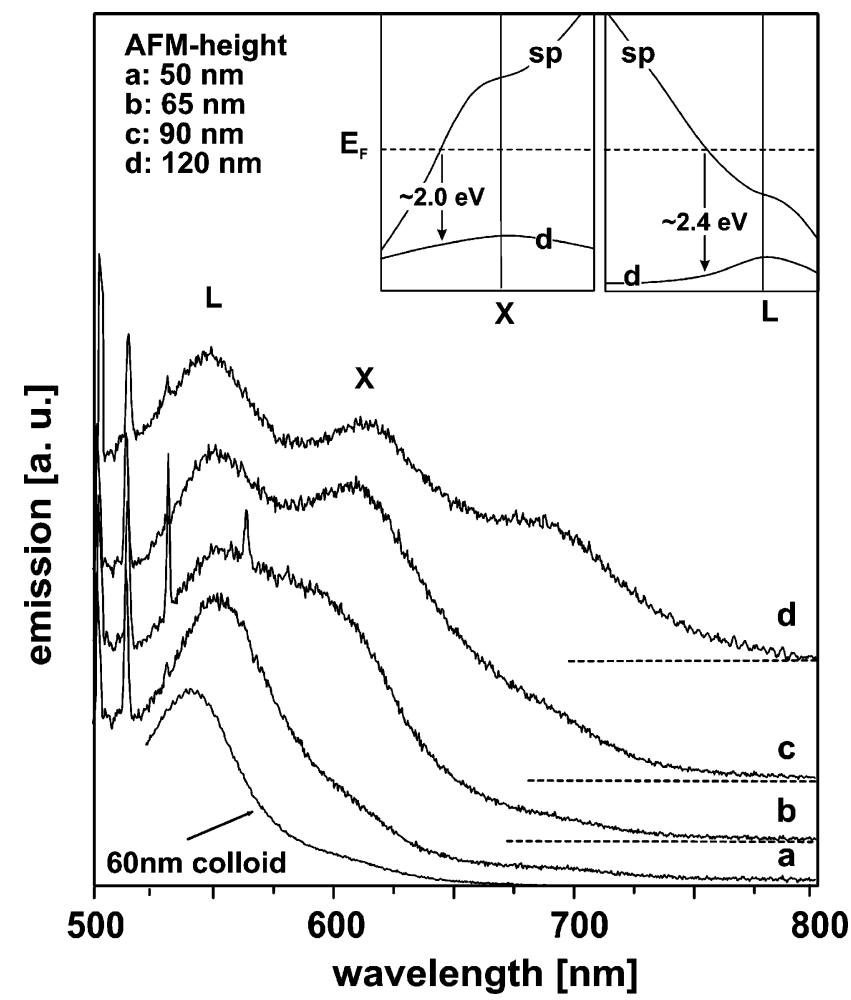

Figure 3. Fluorescence spectra obtained from a $60 \mathrm{~nm}$ gold colloid particle deposited on a $\mathrm{SiO}_{2}$ substrate and from four gold nanoparticles of different diameters $(\mathrm{a}-\mathrm{d})$ electrodeposited on a SWCNT $\left(\lambda_{\mathrm{exc}}=\right.$ $488 \mathrm{~nm}$ ); spectra $\mathrm{b}-\mathrm{d}$ are vertically shifted for clarity, and their relative baselines are indicated. The inset schematically depicts the band structure of gold: radiative recombinations occur close to the $\mathrm{X}$ - and L-symmetry points.

to the nanotubes for 10-30 s generally yielded gold NPs with sizes between 10 and $120 \mathrm{~nm}$. Most likely, gold NPs nucleate at defect positions of the nanotube sidewalls. This observation is supported by the fact that at the end of a CNT, where defects are more likely, metal nanoparticles have been always observed. 


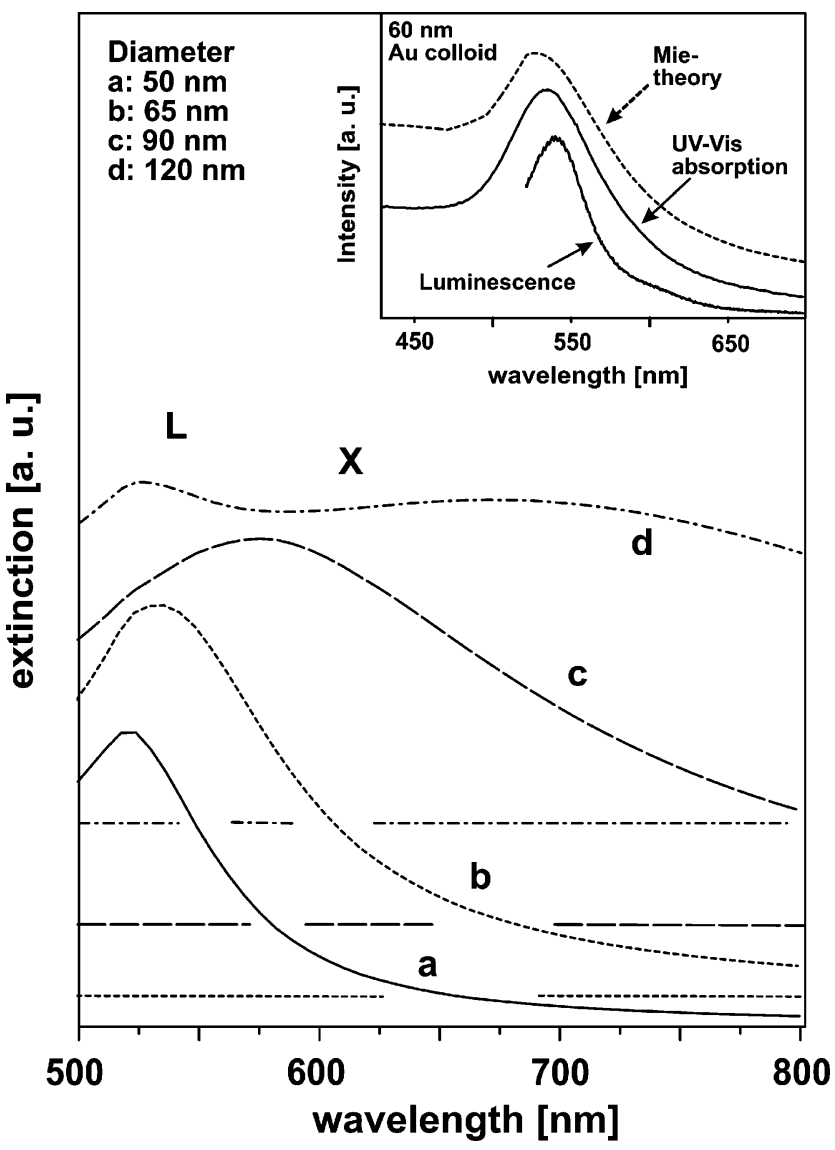

Figure 4. Optical extinction spectra of differently sized gold NPs calculated by using the Mie theory. Spectra $b-d$ are vertically shifted for clarity, and their relative baselines are indicated with corresponding patterns. In the inset, experimental (UV-vis absorption and luminescence) and calculated (Mie theory) spectra of colloidal gold particles are compared: both exhibit a distinct plasmon resonance at the energetic position of the fluorescence transition close to the L-point.

However, more investigations have to be performed to properly address and control the NP densities and nucleation positions.

The optical studies were performed on nanotubes covered with nonaggregated NPs, using a home-built confocal microscope operated under ambient conditions. ${ }^{10}$ For imaging and localization, the sample was scanned through the focus of a high numerical aperture objective (Zeiss Epiplan Apochromat 150X, NA $=0.95$ ), while the emitted light was collected with the same objective and guided through a monochromator to an Avalanche photodiode (EG\&G, SPCM AQR-14). Raman images were recorded by detecting the Raman G-line at $1595 \mathrm{~cm}^{-1}$. Optical spectra were acquired by positioning a SWNT/NPstructure within the diffraction-limited laser spot $\left(\varnothing \approx \lambda_{\text {exc }} / 2\right)$ and utilizing a CCD camera as the detector (PIXIS400B, Princeton Instruments, cooled at $-75{ }^{\circ} \mathrm{C}$ ). Laser powers of $0.1-1 \mathrm{~mW}$ at the entrance of the microscope objective and integration times between 1 and $300 \mathrm{~s}$ were used. By using the electrode as markers, the same tube sections could be found back before and after gold particle decoration.

\section{Results and Discussion}

The effect of gold NP decoration on the spectroscopic response of SWNTs is exemplified in Figure 1, which displays an AFM image (left) and a Raman image (right) acquired from a loop-shaped SWNT decorated with gold NPs of a relatively high density (average separation of 50-100 nm). The Raman
TABLE 1: Comparison of the Measured Raman G-Line Intensity after Background Correction at Three Different Positions along the SWNT in Figure $5 b^{a}$

\begin{tabular}{llllr}
\hline & \multicolumn{4}{c}{ excitation wavelength $(\mathrm{nm})$} \\
\cline { 2 - 5 } position & 488 & 514 & 568 & 647 \\
\hline $1:$ pristine & 16.2 & 3.3 & 47.2 & 1.0 \\
$2: \varnothing_{\mathrm{NP}}=35 \mathrm{~nm}$ & 20.5 & 4.3 & 90.1 & 1.0 \\
$3: \varnothing_{\mathrm{NP}}=70 \mathrm{~nm}$ & 18.5 & 2.3 & 739.5 & 36.6
\end{tabular}

${ }^{a}$ Values are normalized to the silicon substrate peak and related to the G-line intensity at position 1 excited with $647 \mathrm{~nm}$.

image, acquired at $\lambda_{\text {exc }}=568 \mathrm{~nm}$, clearly exhibits bright spots at the locations of some NPs. In Figure 2, optical spectra recorded over a SWNT modified with a NP with a size of 90 $\mathrm{nm}$ (as determined from the AFM height profile) are shown for four different laser wavelengths. Spectrum a in Figure 2, obtained upon excitation with $488 \mathrm{~nm}$, reveals an intense broad feature that is well-fitted by three Lorentzian peaks centered at 546, 617, and $678 \mathrm{~nm}$, respectively. Superimposed on this feature are two narrow peaks at $529.9 \mathrm{~nm}$ (G-line) and 562.7 $\mathrm{nm}\left(\mathrm{G}^{\prime}\right.$-line). These peaks shift with the excitation energy, as can be seen from spectra $b-d$ in Figure 2, whereas the background feature remains unaltered (not shown for clarity). On the basis of these observations, the background signal is ascribed to the fluorescence of the gold particle, and the sharp peaks are attributed to Raman signals originating from the SWNTs.

The fluorescence of gold, discovered in $1969,{ }^{19}$ is explained as a three-step process that involves (i) the photoexcitation of an electron from an occupied d-band to an empty sp-band above the Fermi level, (ii) the relaxation of the electron in the spband to the Fermi level with concomitant scattering of the hole in the d-band to a similar $k$-value as the electron, and (iii) the recombination of the electron-hole pair leading to photon emission. ${ }^{19,20,25}$ Hence, the energy of the emitted photons equals the energetic separation between the Fermi surface and the $\mathrm{d}$-band at $k$-values where the sp-band crosses the Fermi surface (see inset in Figure 3). According to band structure calculations, ${ }^{26}$ these crossing points are located close to the L- and $\mathrm{X}$-symmetry points of the reciprocal space, with respective transition energies of $\sim 2.4 \mathrm{eV}(\sim 515 \mathrm{~nm})$ for the L-point and $\sim 2.0 \mathrm{eV}(\sim 617 \mathrm{~nm})$ for the X-point. Such transitions have already been reported for gold films, ${ }^{20}$ gold nanoparticles, ${ }^{27}$ and gold nanorods. ${ }^{28}$ Here, we assign the first Lorentzian-fitted peak of Figure $2(546 \mathrm{~nm}, \sim 2.3 \mathrm{eV})$ to radiative electron-hole recombination close to the L-point and the second fitted peak $(617 \mathrm{~nm}, \sim 2.0 \mathrm{eV})$ to the recombination close to the X-point. The origin of the third peak around $700 \mathrm{~nm}$ has not been clarified yet. However, a fluorescence band in a similar wavelength range was also observed from other gold species where intraband transitions or surface adsorbates have been put forward as possible explanations. ${ }^{17,27}$

Contrary to the emission energies, the relative intensity of the fluorescence components shows a pronounced size dependence. Figure 3 displays a set of fluorescence spectra obtained from several individual gold particles with different sizes on top of different SWNTs upon excitation with $488 \mathrm{~nm}$. For comparison, the fluorescence spectrum of an individual commercial colloidal gold nanoparticle with a diameter of $60 \mathrm{~nm}$ was also measured. The latter shows a predominant peak at the L-transition energy, while only a weak shoulder occurs at the $\mathrm{X}$-transition. For the nanotube-supported gold particles (spectra $a-d$ in Figure 3), the relative intensity of the X-point transition is seen to increase with particle size. This finding can be 

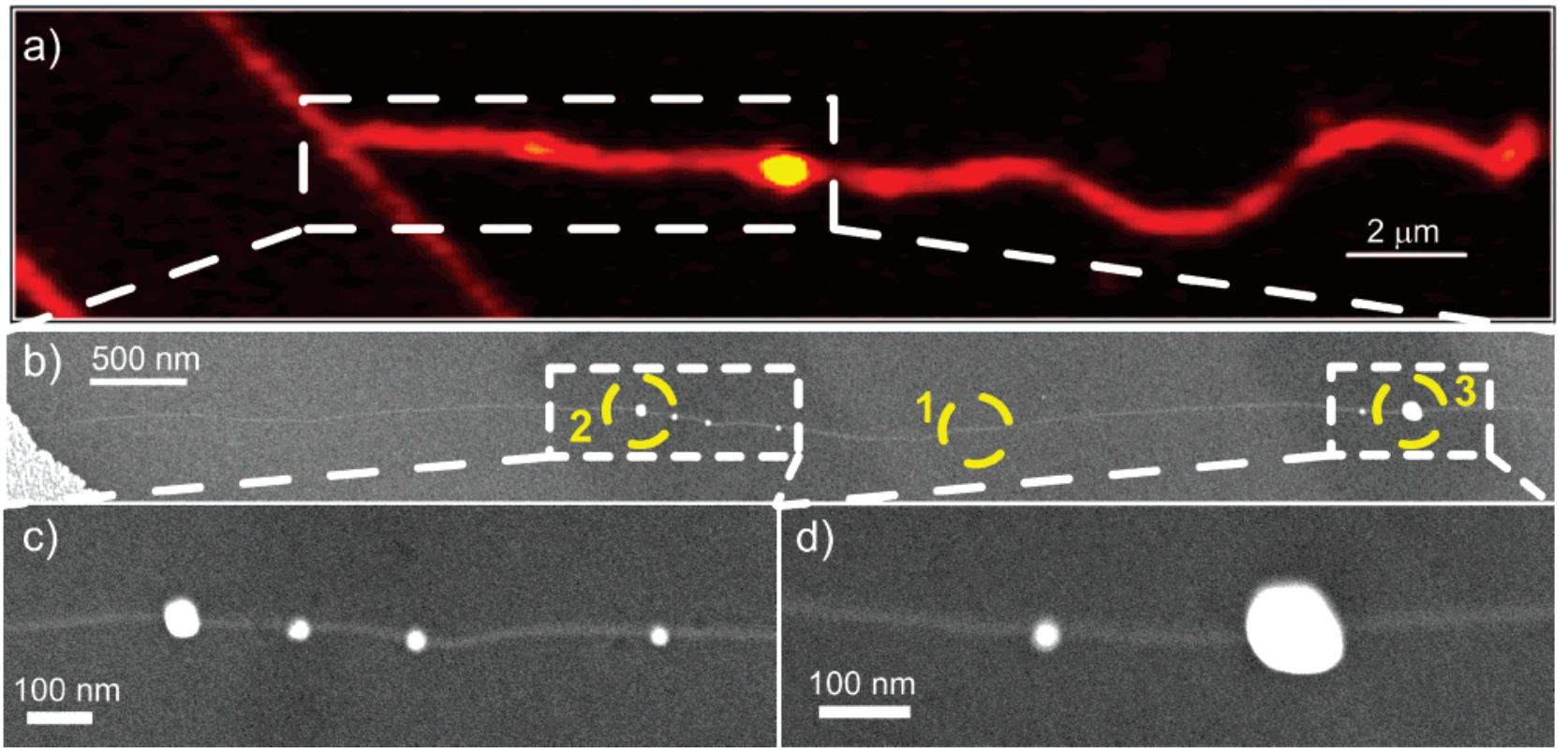

Figure 5. Confocal Raman (a) and SEM (b-d) images of an individual SWNT decorated with gold nanoparticles. The Raman image (a), obtained with $\lambda_{\mathrm{exc}}=568 \mathrm{~nm}$ by detecting the G-line at $1595 \mathrm{~cm}^{-1}$, displays two enhanced regions that are marked by a dashed rectangle. (b) SEM image of the magnified region shows the presence of isolated gold particles. The circles labeled as 1-3 mark the positions where Raman spectra were taken. Position 1 represents a bare section of the tube, while in positions 2 and 3, the tube is decorated by gold nanoparticles. In positions 2 and 3 , the contribution to the Raman enhancement from the neighboring small NPs is negligible. (c and d) SEM images taken at position 2 (NP $\sim 35$ $\mathrm{nm})$ and position $3(\mathrm{NP} \sim 70 \mathrm{~nm})$, respectively.


Figure 6. (Left) Raman spectra acquired with different wavelengths on a bare tube segment (position 1 in Figure 5). (Right) Raman spectra collected with $\lambda_{\text {exc }}=568 \mathrm{~nm}$ on different positions along the tube of Figure 5 . All spectra have been normalized to the $\mathrm{SiO}_{2}$ substrate peak at $\sim 1000 \mathrm{~cm}^{-1}$

explained on the basis of fluorescence enhancement imparted by resonance between the emission wavelength and the particle plasmon. Theoretical models accounting for the local field enhancement predict that the luminescence intensity is given by the following expression: ${ }^{20,29}$

$$
I \sim|E|^{2}\left[L\left(\omega_{\text {in }}\right)\right]^{2}\left[L\left(\omega_{\text {out }}\right)\right]^{2} \beta
$$

where $E$ is the incident electromagnetic field, $L\left(\omega_{\text {in }}\right)$ is the localfield factor at the incoming wavelength, $L\left(\omega_{\text {out }}\right)$ is the localfield factor at the outgoing wavelength, and $\beta$ is a proportionality constant that includes characteristic bulk gold parameters. ${ }^{30}$ Therefore, there is a strong correlation between the particle plasmon frequency and the intensity of the fluorescence. For example, from the inset in Figure 4, it can be seen that measured plasmon energy of the colloidal gold particles coincides with the fluorescence energy mainly at the L-transition, which is therefore strongly enhanced. Since the extinction spectra of the nanotube-supported gold particles cannot be directly measured, we simulated their extinction spectra for sizes similar as shown in Figure 3 using the electrodynamic Mie theory. ${ }^{16}$ This method generally reproduces the extinction spectra of metal particles very well, as exemplified for the $60 \mathrm{~nm}$ colloidal gold particles in the inset of Figure 4. The calculated extinction spectra of the electrochemically prepared particles are shown in Figure $4 \mathrm{a}-\mathrm{d}$ and exhibit pronounced broadening toward larger wavelengths, in agreement with experimental data. ${ }^{31}$ This effect enhances the coupling with long wavelength components of the intrinsic gold fluorescence, whereby the radiative electron-hole recombination close to the $\mathrm{X}$-point is increased.

The Raman responses obtained from a total of $>100$ investigated gold NPs on different individual SWNTs can be categorized into three different types. The majority of the obtained spectra ( 90\%) displayed well-defined Raman peaks 
closely resembling those of pristine nanotubes (e.g., with RBM, D-, G-, or G'-lines) but only upon excitation below $600 \mathrm{~nm}$. For excitation above $600 \mathrm{~nm}, \sim 50 \%$ of those SWNT/NP hybrids showed pronounced spectral diffusion and/or blinking. These phenomena have been frequently encountered in SERS studies on small molecules and are usually attributed to local reorientations of the probed molecules. ${ }^{24}$ Such processes, however, are rather unlikely to occur in the case of bulky SWNTs, and the signal might originate from residuals of the PVP on the surface of the gold NPs. A third type of Raman response has been observed from a very small number $(\sim 10 \%)$ of SWNT/NP hybrids, which exhibited strongly broadened peaks. Since fast spectral fluctuations on the millisecond time scale could be ruled out as a reason for this broadening, this behavior might be due to the presence of amorphous carbon, ${ }^{32}$ which might be produced during the CVD process and could have served as a nucleation site for the NP deposition.

To compare different enhancements of the Raman peaks for different excitation wavelengths, we focused on stable SWNT/ NP hybrid spectra that closely resembled the Raman features of pristine tubes, except for the intensity. A typical example is shown in the Raman image of Figure 5, where a NP-decorated SWNT exhibits a uniform Raman spectrum along its entire length of $\sim 20 \mu \mathrm{m}$. This tube allows investigating the effect of differently sized gold particles on the same nanotube by comparing the spectral features of a bare tube segment (position 1) with two different segments (positions 2 and 3), which are covered by isolated particles of different sizes $(35$ and $70 \mathrm{~nm}$, respectively). A selection of G-line spectra taken with different wavelengths at the bare tube segment is shown in the left panel of Figure 6, where all spectra are normalized to the $\mathrm{SiO}_{2}$ substrate peak at $\sim 1000 \mathrm{~cm}^{-1}$.

As can be seen from Table 1, the spectra collected at position 1 exhibit considerably stronger (up to 47-fold) Raman intensity upon excitation with $568 \mathrm{~nm}$, in comparison to smaller (488 and $514 \mathrm{~nm})$ and larger $(647 \mathrm{~nm})$ wavelengths. This intensity dependence reflects the well-known resonant Raman scattering (RRS) effect occurring when the absorbed or scattered photon is in resonance with an optical transition between the van Hove singularities of the tube. ${ }^{33}$

It is furthermore apparent from Table 1 that for excitation with $568 \mathrm{~nm}$, which ensures resonance with the optical transition of the nanotube, the presence of both particles leads to a significantly enhanced Raman signal. This additional enhancement upon particle decoration stems from the SERS effect (i.e., the local field effect arising from the excited surface plasmon). ${ }^{34}$ The resonant and surface enhancement combine to surfaceenhanced resonance Raman scattering (SERRS), ${ }^{35}$ which is most pronounced for the case of $568 \mathrm{~nm}$ excitation over the $70 \mathrm{~nm}$ particle (see right panel of Figure 6). Under the same conditions, also the particle fluorescence is found to be the most intense. Upon excitation with $568 \mathrm{~nm}$, the larger particle $(\sim 70 \mathrm{~nm})$ leads to stronger Raman and luminescence intensities than the smaller one $(\sim 35 \mathrm{~nm})$ because only for larger particles is the plasmon resonance broadened toward lower energies. ${ }^{31}$ A strong Raman enhancement is therefore also observed for excitation by 647 $\mathrm{nm}$, although in this case, the Raman signal of the unmodified tube is relatively weak because the excitation energy is not in resonance with an electronic transition of the tube, and the plasmon absorption of the $35 \mathrm{~nm}$ particle is too weak to induce a detectable Raman enhancement (see also Supporting Information).

To quantify the Raman enhancement induced by the particles, two factors must be taken into account. First, the Raman signal can already be strong due to the RRS effect. For example, the Raman enhancement factor due to the $70 \mathrm{~nm}$ particle is twice as high at $647 \mathrm{~nm}(\sim 37000)$ as for $568 \mathrm{~nm}(\sim 16000)$, while the absolute Raman intensity at $647 \mathrm{~nm}$ is only $\sim 2 \%$ of the intensity upon $568 \mathrm{~nm}$ excitation. Second, it has to be considered that the local electric field decays very rapidly from the particle surface with a decay length of just a few nm. ${ }^{36}$ Hence, small molecules entirely localized within the near field of the particle experience an increase of the Raman cross-section by many orders of magnitude. ${ }^{37}$ By contrast, only a short segment of the investigated CNT is within the SERS active region close to the particles surface, the length of which should also depend on the detailed particle morphology. In any case, the effective enhancement factor for this tube segment is expected to be at least 1 order of magnitude higher than the factor derived from the observed Raman spectra. It is noteworthy that even stronger local electric field enhancements can occur in nanogaps between adjacent gold particles. ${ }^{38}$ This effect was also observed on the present SWNTs decorated with gold NP aggregates, which is the subject of a separate study. ${ }^{39}$

None of the Raman spectra recorded from the SWNT/NP hybrids displayed sizable shifts (exceeding $\pm 3 \mathrm{~cm}^{-1}$ ) in the energetic position of the D- and $\mathrm{G}^{\prime}$-bands of the nanotubes. This result is in contrast to earlier investigations of SWNTs deposited onto rough silver colloid substrates, ${ }^{40}$ in which shifts of up to 8 and $24 \mathrm{~cm}^{-1}$ were detected for the D- and $\mathrm{G}^{\prime}$-bands, respectively. The absence of such shifts, which have been interpreted as evidence for charge transfer between nanotubes and NPs, indicates that for the present samples, charge transfer plays only a minor role in the Raman enhancement. The predominantly electromagnetic origin of the enhancement is further confirmed by the observation that SWNTs decorated by preformed gold colloid particles $(60 \mathrm{~nm})$ via attachment in solution did not show any enhanced Raman signals. Obviously, the direct growth of metal particles onto the carbon framework of the tubes enables a more intimate physical contact with the NPs.

\section{Conclusion}

In conclusion, we have demonstrated that the plasmon resonance of gold nanoparticles electrodeposited onto SWNTs effectively enhances both the particles' fluorescence and the local Raman response of the tubes. A correlation has been observed between the measured fluorescence peaks and the simulated plasmon resonances for gold NPs of different sizes. The SERS of different SWNTs exhibited a predominant electromagnetic enhancement, which has been found to be strongest when both the optical transition in the nanotube as well as the surface plasmon of the gold particle are in resonance with the excitation wavelength (SERRS). Future studies using nanotubes decorated by different metal particles are expected to provide valuable insight into the extent to which additional factors such as charge transfer between nanoparticles and nanotubes might contribute to the Raman enhancement.

Acknowledgment. This work was supported by the Deutsche Forschungsgemeinschaft (DFG Grants BU1125/3 and ME1380/ 9). We thank Dr. Herbert Kneppe and Gregor Schulte for helpful discussions and technical support.

Supporting Information Available: Fluorescence spectra and AFM height profile for gold nanoparticles. This material is available free of charge via the Internet at http://pubs.acs.org.

\section{References and Notes}

(1) Burghard, M. Surf. Sci. Rep. 2005, 58 (1-4), 1-109. 
(2) Ebbesen, T. W.; Lezec, H. J.; Hiura, H.; Bennett, J. W.; Ghaemi,

H. F.; Thio, T. Nature (London, U.K.) 1996, 382 (6586), 54-56.

(3) Lee, R. S.; Kim, H. J.; Fischer, J. E.; Thess, A.; Smalley, R. E. Nature (London, U.K.) 1997, 388 (6639), 255-257.

(4) Bahr, J. L.; Tour, J. M. J. Mater. Chem. 2002, 12 (7), 1952-1958.

(5) Quinn, B. M.; Dekker, C.; Lemay, S. G. J. Am. Chem. Soc. 2005,

127 (17), 6146-6147.

(6) Wildgoose, G. G.; Banks, C. E.; Compton, R. G. Small 2006, 2 (2), 182-193.

(7) Kong, J.; Franklin, N. R.; Zhou, C. W.; Chapline, M. G.; Peng, S. Cho, K. J.; Dai, H. J. Science (Washington, DC, U.S.) 2000, 287 (5453), $622-625$.

(8) Yao, Z.; Postma, H. W. C.; Balents, L.; Dekker, C. Nature (London, U.K.) 1999, 402 (6759), 273-276.

(9) Bockrath, M.; Cobden, D. H.; McEuen, P. L.; Chopra, N. G.; Zettl, A.; Thess, A.; Smalley, R. E. Science (Washington, DC, U.S.) 1997, 275 (5308), 1922-1925.

(10) Mews, A.; Koberling, F.; Basche, T.; Philipp, G.; Duesberg, G. S.;

Roth, S.; Burghard, M. Adv. Mater. 2000, 12 (16), 1210-1214.

(11) Hartschuh, A.; Pedrosa, H. N.; Novotny, L.; Krauss, T. D. Science (Washington, DC, U.S.) 2003, 301 (5638), 1354-1356.

(12) Vitali, L.; Burghard, M.; Schneider, M. A.; Liu, L.; Wu, S. Y.; Jayanthi, C. S.; Kern, K. Phys. Rev. Lett. 2004, 93 (13), 1361031-1361034.

(13) Li, Y. M.; Kim, W.; Zhang, Y. G.; Rolandi, M.; Wang, D. W.;

Dai, H. J. J. Phys. Chem. B 2001, 105 (46), 11424-11431.

(14) Jiang, C. Y.; Li, J. X.; Zhao, J. L.; Kolb, U.; Mews, A. Nano Lett.

2002, 2 (11), 1209-1213.

(15) Reinhard, B. M.; Siu, M.; Agarwal, H.; Alivisatos, A. P.; Liphardt, J. Nano Lett. 2005, 5 (11), 2246-2252.

(16) Kreibig, U.; Vollmer, M. Optical Properties of Metal Clusters; Springer: Berlin, 1995.

(17) Eustis, S.; El-Sayed, M. J. Phys. Chem. B 2005, 109 (34), 1635016356

(18) Van Dijk, M. A.; Lippitz, M.; Orrit, M. Acc. Chem. Res. 2005, 38 (7), 594-601.

(19) Mooradian. Phys. Rev. Lett. 1969, 22 (5), 185-187.

(20) Boyd, G. T.; Yu, Z. H.; Shen, Y. R. Phys. Rev. B 1986, 33 (12), 7923-7936.

(21) Mohamed, M. B.; Volkov, V.; Link, S.; El-Sayed, M. A. Chem. Phys. Lett. 2000, 317 (6), 517-523.
(22) Varnavski, O. P.; Goodson, T.; Mohamed, M. B.; El-Sayed, M. A Phys. Rev. B 2005, 72 (23), 2354051-2354059.

(23) Nie, S. M.; Emery, S. R. Science (Washington, DC, U.S.) 1997, 275 (5303), 1102-1106.

(24) Maruyama, Y.; Ishikawa, M.; Futamata, M. J. Phys. Chem. B 2004, 108 (2), 673-678.

(25) Dulkeith, E; Niedereichholz, T; Klar, T. A; Feldmann, J; von Plessen, G.; Gittins, D. I.; Mayya, K. S.; Caruso, F. Phys. Rev. B 2004, 70 (20), 2054241-2054244.

(26) Guerrisi, M.; Rosei, R.; Winsemius, P. Phys. Rev. B 1975, 12 (2), $557-563$.

(27) Beversluis, M. R.; Bouhelier, A.; Novotny, L. Phys. Rev. B 2003 , 68 (11), 1154331-11543310.

(28) Imura, K.; Nagahara, T.; Okamoto, H. J. Phys. Chem. B 2005, 109 (27), 13214-13220.

(29) Apell, P.; Monreal, R.; Lundqvist, S. Phys. Scr. 1988, 38 (2), 174179.

(30) Chen, C. K.; Heinz, T. F.; Ricard, D.; Shen, Y. R. Phys. Rev. B 1983, 27 (4), 1965-1979.

(31) Sonnichsen, C.; Franzl, T.; Wilk, T.; von Plessen, G.; Feldmann, J. New J. Phys. 2002, 4, 93.1-93.8.

(32) Veres, M.; Fule, M. Toth, S.; Koos, M.; Pocsik, I. Diamond Relat. Mater. 2004, 13 (4-8), 1412-1415.

(33) Reich, S.; Thomsen, C.; Maultzsch, J. Carbon Nanotubes; Wiley VCH: Weinheim, Germany, 2004.

(34) Kneipp, K.; Kneipp, H. Appl. Spectrosc. 2006, 60 (12), 322-334.

(35) Kneipp, K.; Jorio, A.; Kneipp, H.; Brown, S. D. M.; Shafer, K.; Motz, J.; Saito, R.; Dresselhaus, G.; Dresselhaus, M. S. Phys. Rev. B 2001, $6308(8), 814011-814014$.

(36) Hao, E.; Schatz, G. C. J. Chem. Phys. 2004, 120 (1), 357-366.

(37) Otto, A.; Mrozek, I.; Grabhorn, H.; Akemann, W. J. Phys.: Condensed Matter 1992, 4 (5), 1143-1212.

(38) Imura, K.; Okamoto, H.; Hossain, M. K.; Kitajima, M. Nano Lett. 2006, 6 (10), 2173-2176.

(39) Assmus, T.; Balasubramanian, K.; Burghard, M.; Kern, K.; Scolari, M.; Nan, F.; Myalitsin, A.; Mews, A. Appl. Phys. Lett. 2007, 90, 173109

(40) Corio, P.; Brown, S. D. M.; Marucci, A.; Pimenta, M. A.; Kneipp, K.; Dresselhaus, G.; Dresselhaus, M. S. Phys. Rev. B 2000, 61 (19), 1320213211. 